2005

\title{
Stability of stratified flow with inhomogeneous shear
}

\author{
Vladimir S. Mikhailenko
}

Earl E. Scime

Vladimir V. Mikhailenko

Follow this and additional works at: https://researchrepository.wvu.edu/faculty_publications

\section{Digital Commons Citation}

Mikhailenko, Vladimir S.; Scime, Earl E.; and Mikhailenko, Vladimir V., "Stability of stratified flow with inhomogeneous shear" (2005). Faculty Scholarship. 345.

https://researchrepository.wvu.edu/faculty_publications/345 


\title{
Stability of stratified flow with inhomogeneous shear
}

\author{
Vladimir S. Mikhailenko* \\ Kharkov National University, 61108 Kharkov, Ukraine and National Science Center "Kharkov Institute of Physics and Technology," \\ Scientific and Production Complex "Renewable energy sources and sustainable technologies," 61108 Kharkov, Ukraine \\ Earl E. Scime \\ Physics Department, West Virginia University, Morgantown, West Virginia, 26506, USA
}

Vladimir V. Mikhailenko

Kharkov National University, 61108 Kharkov, Ukraine

(Received 13 July 2004; published 11 February 2005)

\begin{abstract}
The temporal evolution of perturbations in stratified flow with inhomogeneous shear is examined analytically by an extension of the nonmodal approach to flows with inhomogeneous shear. The solutions of the equations that govern the linear evolution and the weak nonlinear evolution of perturbations of the stream function for stratified flow with monotonic inhomogeneous shear are obtained. It is shown that stabilization of perturbations arises from nonmodal effects due to flow shear. Conditions at which these nonmodal effects may be strong enough to stabilize the Rayleigh-Taylor instability are presented. These analytical results are also compared to numerical simulations of the governing equations performed by Benilov, Naulin, and Rasmussen.
\end{abstract}

DOI: 10.1103/PhysRevE.71.026306

\section{INTRODUCTION}

It is well known that inversely stratified fluids for which density increases upward are unstable due to the RayleighTaylor instability for all wavelengths [1]. The RayleighTaylor instability in a plasma is governed by similar equations for perturbations of electrostatic potential and density. That instability develops in plasmas embedded in an unfavorably curved magnetic field with a density gradient antiparallel to the magnetic field radius of curvature. In such magnetically confined plasmas, examples of Rayleigh-Taylor modes include the ideal and resistive balooning instabilities [2]. A considerable amount of research has been devoted to the study of the Rayleigh-Taylor instability in fluids and its application to ionospheric turbulence. It is believed that the Rayleigh-Taylor instability can play a major role in the onset of equatorial spread F $[3,4]$. In these physical systems, the essential characteristics of the Rayleigh-Taylor instability are similar. The instability arises in inhomogeneous media (fluid or plasma) and acts to interchange high- and low-density regions or to interchange high- and low-temperature regions.

In many regions of interest, such as the atmosphere, the ionosphere, or the edge of tokamak plasmas, ordinary fluids or plasma may also contain inhomogeneously sheared flows, i.e., flow fields with a spatially varying flow speed gradient. It has been shown that homogeneous velocity shear can have a dramatic effect on the Rayleigh-Taylor instability, e.g., Miles [5] and Kuo [6] demonstrated that flow shear can suppress the Rayleigh-Taylor instability in unbound systems with homogeneously sheared flows. Suppression of the Rayleigh-Taylor instability in plasmas by shear flow was also demonstrated in linear theory by Guzdar et al. [7]. Significant stabilization occurs in the linear theory for $\left|v_{0}^{\prime}(z)\right|$ $\geqslant 2^{-1 / 2} \gamma$, where $v_{0}^{\prime}(z)$ is the velocity shear and the growth

\footnotetext{
*Electronic address: vmikhailenko@kipt.kharkov.ua
}

PACS number(s): 47.20.-k, 47.10.+g, 52.35.Kt

rate of the instability in the absence of flow shear is $\gamma$.

Historically, two different approaches have been used in the development of linear stability theories for systems with sheared flows. Both techniques employ a spectral expansion in time. The first is the method of normal modes or the "modal" approach. In a system with a $z$-dependent flow velocity $v_{0}(z)$ directed along the $x$ axis, the perturbations are assumed to be harmonic in time with separable time and space dependencies described by $\psi(\mathbf{r}, t)=\psi(z) \exp (-i \omega t$ $+i k x)$, where $\psi(z)$ defines the mode structure. The flow is deemed unstable if at last one mode grows exponentially with time. In the case of an ordinary sheared fluid, the mode structure $\psi(z)$ is governed by a second-order differential equation that possesses a singularity at a critical level [9] where the Doppler-shifted phase velocity $(\omega / k)-v_{0}(z)$ vanishes. Because of the singularity, the equations governing the modal structure are non-normal, i.e., the eigenfunctions associated with the governing differential equation are not mutually orthogonal and experience strong interference. Therefore, a stability analysis based on considering the only eigenvalues, $\omega$, obtained from the modal approach may be inappropriate for certain ranges of system parameters, and a solution based on solving the initial value problem is preferred. Several authors have pursued solutions of the initial value problem through the use of a Laplace transform in time. The principal finding of the initial-value problem approach is that, in addition to the discrete eigenvalues linked to the normal modes, there exists a continuous spectrum of eigenvalues. Thus, the modal approach cannot provide a complete solution for all sheared flow systems. It was demonstrated (see, for example, Ref. [8]) that owing to the existence of the continuous spectrum of eigenvalues, initial disturbances may decay or even grow as a nonmodal perturbation with nonseparable space and time dependencies and with time-dependent amplitudes that are power-law functions of time. Such disturbances, at certain times, may overwhelm 
the exponentially growing modes of the discrete spectrum [12] and always dominate over the exponentially decaying modes. Therefore, for the conditions at which the RayleighTaylor instability is stabilized by sheared flow, nonmodal effects should be considered. For those time intervals during which the nonmodal solutions dominate, the nonmodal effects may even become strong enough to disrupt the development of the typical nonlinear processes predicted by nonlinear theories of normal mode evolution, and therefore new nonlinear processes due to the growth of the nonmodal solutions should also be considered.

An alternative approach to the initial-value problem solution for unbounded, homogeneously sheared flow was described by Hartman [13]. That approach involves a transformation to coordinates in the local rest frame of the flow and does not invoke the normal mode ansatz or any spectral expansion in time. This method, previously used by Lord Kelvin [14] in studying the evolution of initial disturbances in parallel viscous flows with uniform shear, and by Phillips [15] in investigations of internal waves in a weakly sheared thermocline, yielded a successful analysis of the evolution of disturbances of the flows of fluids and plasmas with homogeneous shear (see, for example, Refs. [17,18] and references therein). In the flow frame coordinates, the evolution of an initial perturbation in homogeneously sheared flow is solvable analytically for any time of interest and is free from ambiguities arising from the mathematical singularity appeared at a normal-mode critical level. Hartman's analysis obtains the same constraint, $\left|v_{0}^{\prime}(z)\right| \geqslant 2^{-1 / 2} \gamma$ on the velocity shear $v_{0}^{\prime}(z)$ for stabilization and suppression of RayleighTaylor instability as was obtained with the modal approach. However, Hartman found that the solution to the initial-value problem has a typically nonmodal power-law temporal dependence and thus the normal mode solution is not the steady-state limit for the initial-value problem. Weak nonlinear analysis of the Rayleigh-Taylor instability in a plasma, performed by Mikhailenko et al. [17] using a nonmodal approach, demonstrated that homogeneous flow shear stabilizes not only linearly unstable two-dimensional perturbations of electrostatic potential, but also nonlinearly unstable (terms including the fourth order of the perturbed potential were considered) perturbations. Compared to the previous linear analyses, the nonlinear analysis yielded a slightly different constraint on the magnitude of the velocity shear required for stabilization of the Rayleigh-Taylor instability.

Until now, the nonmodal approach has only been applied to systems with homogeneous flow shear, i.e., $v_{0}^{\prime}(z)$ independent of $z$. In this work, we develop an analytic framework for the extension of the nonmodal approach to flows with inhomogeneous shear and we also examine the stability of stratified flows with inhomogeneous shear. We will consider at the outset only two-dimensional perturbations in a stratified medium. The application of a two-dimensional model is justified by the two-dimensional nature of the Rayleigh-Taylor instability in fusion [2] and ionospher [7] plasma as well as in internal gravity waves in ordinary stratified fluids $[10,11]$. The structure of this paper is as follows. In the next section, we formulate the basic nonmodal nonlinear equation that can be solved asymptotically in the case of inhomogeneous flow shear. In Sec. III, the solutions of that equation for stratified flows with inhomogeneous, monotonic, velocity shear are developed. In Sec. IV, the weak nonlinear temporal evolution of the nonmodal solutions is studied and some concluding remarks are presented in Sec. V.

\section{THE GOVERNING EQUATIONS}

We choose as our model a two-dimensional unbounded plane with inhomogeneously sheared flow and an exponential density scaling. The equilibrium velocity, pressure, and density are given by $\mathbf{v}_{0}=v_{0}(z) \mathbf{e}_{x}, P(z)$ $=P_{0} \exp \left[-g \int^{z} \rho_{0}\left(z_{1}\right) d z_{1}\right]$, and $\rho_{0}(z)=\rho_{00} \exp (-z / H)$, where $g$ is the acceleration due to gravity, $\rho_{00}$ is the mean density, and $H$ is the scale height for the density. The fluid is assumed to be incompressible and therefore a stream function, $\psi=\psi \mathbf{e}_{y}$, can be defined through

$$
\mathbf{v}=\boldsymbol{\nabla} \psi=\left(-\frac{\partial \psi}{\partial z}, \frac{\partial \psi}{\partial x}\right) .
$$

The solution of the temporal evolution of inhomogeneously sheared flow reduces to the solution of the set of differential equations for $\psi$ and density perturbation, $\rho$,

$$
\begin{aligned}
\left(\frac{\partial}{\partial t}+\right. & \left.v_{0}(z) \frac{\partial}{\partial x}\right)(-\Delta \psi)+v_{0}^{\prime \prime}(z) \frac{\partial \psi}{\partial x}+\left(\frac{\partial \psi}{\partial z} \frac{\partial}{\partial x}-\frac{\partial \psi}{\partial x} \frac{\partial}{\partial z}\right) \Delta \psi \\
= & \frac{g}{\rho_{0}} \frac{\partial \rho}{\partial x} \\
& \left(\frac{\partial}{\partial t}+v_{0}(z) \frac{\partial}{\partial x}-\frac{\partial \psi}{\partial z} \frac{\partial}{\partial x}+\frac{\partial \psi}{\partial x} \frac{\partial}{\partial z}\right) \rho=N^{2} \frac{g}{\rho_{0}} \frac{\partial \psi}{\partial x},
\end{aligned}
$$

where the prime denotes $d / d z$. The linearized version of Eqs. (1) and (2) may be combined through the Boussinesq approximation into the equation

$$
\left(\frac{\partial}{\partial t}+v_{0}(z) \frac{\partial}{\partial x}\right)\left[\left(\frac{\partial}{\partial t}+v_{0}(z) \frac{\partial}{\partial x}\right) \Delta \psi-v_{0}^{\prime \prime}(z) \frac{\partial \psi}{\partial x}\right]=-N^{2} \frac{\partial^{2} \psi}{\partial x^{2}},
$$

in which

$$
N^{2}=-\frac{g \rho_{0}^{\prime}(z)}{\rho_{0}}
$$

is the Brunt-Väisälä frequency. In the normal mode approach, in which a stream function $\psi$ is assumed to be harmonic in $t$, i.e., $\psi(z) \exp (i k x-i \omega t)$, the equation for the mode structure $\psi(z)$ is called the Taylor-Goldstein equation and has the form

$$
\frac{d^{2} \psi}{d z^{2}}-\left(k^{2}+\frac{k v_{0}^{\prime \prime}(z)}{\left(k v_{0}(z)-\omega\right)}-\frac{k^{2} N^{2}}{\left(k v_{0}(z)-\omega\right)^{2}}\right) \psi=0
$$

with singular points, where the phase velocity matches the equilibrium velocity, $\omega / k-v_{0}(z)=0$. Such points are commonly referred to as critical levels [9]. It follows from Eq. (4) that estimation of the shear-flow effect is related to the solution of the nonlinear eigenvalue problem with singular nonorthogonal eigenfunctions in the modal approach. 
To avoid the mathematical difficulties involved in the normal mode approach, here we use the nonmodal method. Our approach permits the development of asymptotic methods for analysis of the linear as well as the weak nonlinear evolutionary stages for systems in which the inhomogeneous flow shear $v_{0}^{\prime}(z)$ is either a small or a large parameter. The nonmodal approach begins with a transformation to the convective coordinates

$$
\xi=x-v_{0}(z) t, \quad \eta=z, \quad t=t,
$$

that are the coordinates in the local rest frame of the mean flow. These coordinates are generalizations of the convective coordinates used previously in systems with homogeneously sheared flows [13-17]. In terms of the convective coordinates, the system of equations may be combined into a single nonlinear differential equation for the stream function $\psi$

$$
\begin{gathered}
\frac{1}{v_{0}^{\prime}(\eta) T} \frac{\partial}{\partial \tau}\left[\frac{1}{v_{0}^{\prime}(\eta) T} \frac{\partial}{\partial \tau} \Delta \psi-\frac{v_{0}^{\prime \prime}(\eta)}{v_{0}^{\prime}(\eta)} \frac{\partial \psi}{\partial \xi}\right]+J(\eta) \frac{\partial^{2} \psi}{\partial \xi^{2}} \\
=2 \frac{\varepsilon T}{\left[v_{0}^{\prime}(\eta) T\right]^{2}} \frac{\partial}{\partial \tau}\left(\frac{\partial \psi}{\partial \eta} \frac{\partial \Delta \psi}{\partial \xi}-\frac{\partial \psi}{\partial \xi} \frac{\partial \Delta \psi}{\partial \eta}\right) \\
-\frac{\varepsilon T}{\left[v_{0}^{\prime}(\eta) T\right] v_{0}^{\prime}(\eta)}\left(\frac{\partial \psi}{\partial \eta} \frac{\partial}{\partial \xi}-\frac{\partial \psi}{\partial \xi} \frac{\partial}{\partial \eta}\right) v_{0}^{\prime \prime}(\eta) \frac{\partial \psi}{\partial \xi} \\
-\frac{(\varepsilon T)^{2}}{\left[v_{0}^{\prime}(\eta) T\right]^{2}}\left(\frac{\partial \psi}{\partial \eta} \frac{\partial}{\partial \xi}-\frac{\partial \psi}{\partial \xi} \frac{\partial}{\partial \eta}\right)\left(\frac{\partial \psi}{\partial \eta} \frac{\partial}{\partial \xi}-\frac{\partial \psi}{\partial \xi} \frac{\partial}{\partial \eta}\right) \Delta \psi
\end{gathered}
$$

where $J(\eta)=\left[N / v_{0}^{\prime}(\eta)\right]^{2}$ is the Richardson number. Equation (6) contains two parameters. One, $v_{0}^{\prime}(\eta) T$, is the magnitude of the flow shear (shear parameter), and the second, $\varepsilon T$, is an amplitude parameter, which defines a measure of the nonlinearity. $\tau$ is the dimensionless time variable, defined by $t$ $=T \tau$, where $T$ is the time scale of interest. The Laplacian operator $\Delta$ in the new variables is time-dependent and is equal to

$$
\begin{aligned}
\Delta & =\frac{\partial^{2}}{\partial x^{2}}+\frac{\partial^{2}}{\partial z^{2}}, \\
= & \frac{\partial^{2}}{\partial \xi^{2}}+\frac{\partial^{2}}{\partial \eta^{2}}-2\left[v_{0}^{\prime}(\eta) T\right] \tau \frac{\partial^{2}}{\partial \xi \partial \eta}-\frac{v_{0}^{\prime \prime}(\eta)}{v_{0}^{\prime}(\eta)}\left[v_{0}^{\prime}(\eta) T\right] \tau \frac{\partial}{\partial \xi} \\
& +\left[v_{0}^{\prime}(\eta) T\right]^{2} \tau^{2} \frac{\partial^{2}}{\partial \xi^{2}} .
\end{aligned}
$$

In the new differential equation, the shear parameter, $v_{0}^{\prime}(\eta) T$, can be considered to be a small or large asymptotic parameter to determine the qualitative behavior of the solutions and to develop appropriate asymptotic methods for solution in the cases of weak or strong flow shear. Performing a Fourier transformations of Eq. (6) over variable $\xi$,

$$
\psi(\tau, l, \eta)=\int d \xi \exp (-i \xi l) \psi(\tau, \xi, \eta)
$$

the equation for $\psi(\tau, l, \eta)$ is obtained from Eq. (6) and is

$$
\begin{aligned}
& \frac{1}{\left[v_{0}^{\prime}(\eta) T\right]} \frac{\partial}{\partial \tau}\left[\frac{1}{\left[v_{0}^{\prime}(\eta) T\right]} \frac{\partial}{\partial \tau} \Delta \psi(\tau, l, \eta)-i l \frac{v_{0}^{\prime \prime}(\eta)}{v_{0}^{\prime}(\eta)} \psi(\tau, l, \eta)\right]-l^{2} N^{2} \psi(\tau, l, \eta) \\
& \quad=\int d l_{1} \int d l_{2} \delta\left(l-l_{1}-l_{2}\right)\left[2 \frac{\varepsilon T}{\left[v_{0}^{\prime}(\eta) T\right]^{2}} \frac{\partial}{\partial \tau}\left(\frac{\partial \psi\left(\tau, l_{1}, \eta\right)}{\partial \eta} i l_{2} \Delta \psi\left(\tau, l_{2}, \eta\right)-i l_{1} \psi\left(\tau, l_{1}, \eta\right) \frac{\partial}{\partial \eta} \Delta \psi\left(\tau, l_{2}, \eta\right)\right)\right. \\
& \left.\quad-\frac{\varepsilon T}{\left[v_{0}^{\prime}(\eta) T\right] v_{0}^{\prime}}\left(v_{0}^{\prime \prime \prime}(\eta) l_{1} l_{2} \psi\left(\tau, l_{1}, \eta\right) \psi\left(\tau, l_{2}, \eta\right)+v_{0}^{\prime \prime}(\eta) l_{1} l_{2} \psi\left(\tau, l_{1}, \eta\right) \frac{\partial \psi\left(\tau, l_{2}, \eta\right)}{\partial \eta}-v_{0}^{\prime \prime}(\eta) l_{2}^{2} \psi\left(\tau, l_{2}, \eta\right) \frac{\partial \psi\left(\tau, l_{1}, \eta\right)}{\partial \eta}\right)\right],
\end{aligned}
$$

where the terms of the order of $\psi^{3}$ are omitted and

$$
\Delta(\tau, l, \eta)=\frac{\partial^{2}}{\partial \eta^{2}}-2 i l \tau v_{0}^{\prime}(\eta) \frac{\partial}{\partial \eta}-i l v_{0}^{\prime \prime}(\eta) \tau-l^{2}\left[v_{0}^{\prime}(\eta)\right]^{2} \tau^{2}-l^{2}
$$

Equation (9) includes the effects of both flow shear inhomogeneity and nonlinearity. To understand the role both of these effects play in the complete solution, it is instructive to consider these effects separately.

It follows from Eqs. (6) and (7) [or Eqs. (9) and (10)] that flow shear is the source of the nonmodal time dependence of the stream function. Nonmodal effects are negligible in the case of weak flow shear, i.e., $\left|v_{0}^{\prime}(\eta)\right| T \ll 1$. For $T=\gamma^{-1}$, where $\gamma \approx\left|N\left(z_{0}\right)\right|$ is the local growth rate of the ordinary instability of the inversely stratified fluid $[9,19]$ initial perturbations develop as are typically obtained in modal approach solutions. The development of the modal instability is followed by the development of nonlinear effects, which ultimately may lead to instability saturation. Such "modal" turbulence may affect the mean flow by changing its structure through processes such as the formation Kelvin cat-eyes vortices in the critical layer regions [9] and/or development of turbulent viscosity. It is only during the long time evolution of such nonlinear processes that nonmodal effects might be important in the case of weak flow shear.

Is follows from Eq. (10) that nonmodal effects vanish for values $\eta_{0}$ such that $v_{0}^{\prime}\left(\eta_{0}\right)=0$. Nearby these zero shear regions, nonmodal effects are weak and therefore perturbations will develop as governed by the modal equations and the effects of shear flow are minimal. Note that all nonmodal 
terms in Eq. (9) are multiplied by the wave-number component $l$ along the shear flow. Therefore, nonmodal effects also vanish for disturbances with $l=0$. Small values of $l$ reduce the effect of the flow shear and thus nonmodal effects are again minimized. It follows from Eqs. (9) and (10) that nonmodal effects dominate when $\left|v_{0}^{\prime}(\eta) T\right| \geqslant 1$ and the wave number $l$ is not small. Comparison of the modal and nonmodal terms in Eqs. (9) and (10) leads to a general condition for dominance of nonmodal effects,

$$
\left|v_{0}^{\prime}(\eta)\right| T>\max \left(1 ; \frac{\left|v_{0}^{\prime \prime}(\eta)\right|}{\left|l v_{0}^{\prime}(\eta)\right|}, \frac{1}{l h}\right),
$$

where $h$ is the scale length of the perturbation along the flow shear. For $T=\gamma^{-1}$, Eq. (11) becomes

$$
\left|v_{0}^{\prime}(\eta)\right|>\max \left(\gamma ; \gamma \frac{\left|v_{0}^{\prime \prime}(\eta)\right|}{\left|l v_{0}^{\prime}(\eta)\right|}, \gamma \frac{1}{l h}\right) .
$$

When Eq. (12) is satisfied, the initial perturbation develops according to the nonmodal constraints prior to the development of the modal instability. Therefore, Eq. (12) is akin to a bifurcation condition that separates two distinctively different types of solutions to Eq. (9).

The temporal evolution of perturbations in the case of strong, monotonic, inhomogeneous flow shear for which Eq. (12) is satisfied is the focus of the rest of the analytic analysis presented in this work. The temporal evolution of perturbations in the case of monotonic, inhomogeneous flow shear was considered numerically by Benilov et al. [19]. They showed that inhomogeneous flow shear stabilizes only short scale disturbances and leaves unstable large scale disturbances, i.e., those with small wave number $l$. In the next sections, we obtain linear and weak nonlinear solutions to Eq. (9) for inhomogeneous flow shear under the constraint given by Eq. (12) for the dominance of nonmodal effects. We show that under these conditions, the solutions for stream function are stable. Therefore, Eq. (12) defines a boundary for the regions of stable and unstable wave number $l$.

\section{LINEAR EVOLUTION STAGE OF PERTURBATIONS IN INHOMOGENEOUSLY SHEARED FLOW}

Here we consider the effect of inhomogeneous shear on the linear temporal evolution of perturbations in a system with stratified flow. In the case of strong flow shear, it is convenient to introduce a new variable, $\zeta(\tau, \eta, l)$, defined by

$$
\zeta(\tau, \eta, l)=\left[v_{0}^{\prime}(\eta) T \tau\right]^{2} \psi(\tau, \eta, l)
$$

For zero order in the nonlinearity parameter, $\varepsilon T$ [i.e., we omit the right nonlinear part of Eq. (9)], we obtain the following equation for $\zeta$ :

$$
\begin{aligned}
& l^{2} \frac{\partial^{2} \zeta(\tau, l, \eta)}{\partial \tau^{2}}+\frac{N^{2} l^{2}}{\left(v_{0}^{\prime}\right)^{2}} \zeta(\tau, l, \eta)+\frac{2 i l}{\left(v_{0}^{\prime} T\right)} \frac{1}{\tau} \frac{\partial^{3} \zeta}{\partial \tau^{2} \partial \eta}-3 i l\left(\frac{v_{0}^{\prime \prime}}{v_{0}^{\prime}}\right) \frac{1}{\left(v_{0}^{\prime} T\right)} \frac{1}{\tau} \frac{\partial^{2} \zeta}{\partial \tau^{2}}-8 i l \frac{v_{0}^{\prime \prime}}{v_{0}^{\prime}} \frac{1}{\left(v_{0}^{\prime} T\right)} \frac{1}{\tau^{3}} \zeta(\tau, l, \eta)-\frac{4 i l}{\left(v_{0}^{\prime} T\right)} \frac{1}{\tau^{2}} \frac{\partial^{2} \zeta}{\partial \tau \partial \eta}+\frac{4 i l}{\left(v_{0}^{\prime} T\right)} \frac{1}{\tau^{3}} \frac{\partial \zeta}{\partial \eta} \\
& +7 i l\left(\frac{v_{0}^{\prime \prime}}{v_{0}^{\prime}}\right) \frac{1}{\left(v_{0}^{\prime} T\right)} \frac{1}{\tau^{2}} \frac{\partial \zeta}{\partial \tau}+\frac{6 l^{2}}{\left(v_{0}^{\prime} T\right)^{2}} \frac{1}{\tau^{4}} \zeta(\tau, l, \eta)+12 \frac{\partial}{\partial \eta}\left(\frac{v_{0}^{\prime \prime}}{v_{0}^{\prime}} \frac{1}{\left(v_{0}^{\prime} T\right)^{2}}\right) \frac{1}{\tau^{4}} \zeta(\tau, l, \eta)+2 \frac{\partial}{\partial \eta}\left(\frac{v_{0}^{\prime \prime}}{v_{0}^{\prime}} \frac{1}{\left(v_{0}^{\prime} T\right)^{2}}\right) \frac{1}{\tau^{2}} \frac{\partial^{2} \zeta}{\partial \tau^{2}}-\frac{4 l^{2}}{\left(v_{0}^{\prime} T\right)^{2}} \frac{1}{\tau^{3}} \frac{\partial \zeta}{\partial \tau} \\
& \quad-8 \frac{\partial}{\partial \eta}\left(\frac{v_{0}^{\prime \prime}}{v_{0}^{\prime}} \frac{1}{\left(v_{0}^{\prime} T\right)^{2}}\right) \frac{1}{\tau^{3}} \frac{\partial \zeta}{\partial \tau}+24\left(\frac{v_{0}^{\prime \prime}}{v_{0}^{\prime}}\right) \frac{1}{\left(v_{0}^{\prime} T\right)^{2}} \frac{1}{\tau^{4}} \frac{\partial \zeta}{\partial \eta}+4\left(\frac{v_{0}^{\prime \prime}}{v_{0}^{\prime}}\right) \frac{1}{\left(v_{0}^{\prime} T\right)^{2}} \frac{1}{\tau^{2}} \frac{\partial^{3} \zeta}{\partial \eta \partial \tau^{2}}-16\left(\frac{v_{0}^{\prime \prime}}{v_{0}^{\prime}}\right) \frac{1}{\left(v_{0}^{\prime} T\right)^{2}} \frac{1}{\tau^{3}} \frac{\partial^{2} \zeta}{\partial \eta \partial \tau}-\frac{1}{\left(v_{0}^{\prime} T\right)^{2}} \frac{1}{\tau^{2}} \frac{\partial^{4} \zeta}{\partial \eta^{2} \partial \tau^{2}} \\
& +\frac{l^{2}}{\left[v_{0}^{\prime}(\eta) T\right]^{2}} \frac{1}{\tau^{2}} \frac{\partial^{2} \zeta}{\partial \tau^{2}}+\frac{4}{\left(v_{0}^{\prime} T\right)^{2}} \frac{1}{\tau^{3}} \frac{\partial^{3} \zeta}{\partial \eta^{2} \partial \tau}-\frac{6}{\left(v_{0}^{\prime} T\right)^{2}} \frac{1}{\tau^{4}} \frac{\partial^{2} \zeta}{\partial \eta^{2}}=0 .
\end{aligned}
$$

The presence of the small parameter $\left[v_{0}^{\prime}(\eta) T\right]^{-1} \ll 1$ in Eq. (14) permits us to obtain a solution in terms of a power series in the parameter $\left[v_{0}^{\prime}(\eta) T\right]^{-1}$,

$$
\begin{aligned}
\zeta(\tau, l, \eta)= & \zeta_{0}(\tau, l, \eta)+\frac{1}{\left[v_{0}^{\prime}(\eta) T\right]} \zeta_{1}(\tau, l, \eta) \\
& +\frac{1}{\left[v_{0}^{\prime}(\eta) T\right]^{2}} \zeta_{2}(\tau, l, \eta)+\cdots
\end{aligned}
$$

By employing this new power series approach, we can obtain homogeneous asymptotic solutions for times $\tau \geqslant\left[v_{0}^{\prime}(\eta) T\right]^{-1}$ in systems with inhomogeneously sheared flows for which the condition $\left[v_{0}^{\prime}(\eta) T\right]^{-1} \ll 1$ is fulfilled for all considered values of $\eta$. For $\zeta_{0}$, we have the equation

$$
\frac{\partial^{2} \zeta_{0}(\tau, l, \eta)}{\partial \tau^{2}}+J(\eta) \frac{1}{\tau^{2}} \zeta_{0}(\tau, l, \eta)=0,
$$

obtained earlier by Hartman [13] for the case of the homogeneous flow shear. The solution to Eq. (16) is easily obtained and is equal to

$$
\zeta_{0}(\tau, l, \eta)=C_{1}(l, \eta) \tau^{k_{1}}+C_{2}(l, \eta) \tau^{k_{2}}
$$

for the case $J(\eta) \neq 1 / 4$, where

$$
k_{1,2}=\frac{1}{2} \pm \sqrt{\frac{1}{4}-J(\eta)} .
$$

For $J(\eta)=1 / 4$, the solution is

$$
\zeta_{0}(\tau, l, \eta)=\tau^{1 / 2}\left[C_{1}(l, \eta)+C_{2}(l, \eta) \ln \tau\right] .
$$


It follows from the solution (17) that the stream function $\psi$ decays with increasing time, when the condition

$$
\left|v_{0}^{\prime}(\eta)\right| \geqslant 2^{-1 / 2} \gamma
$$

is fulfilled for all $\eta$ considered (as it is in the case of the homogeneous shear flow).

The perturbation of the fluid density in the convective coordinates is given by

$$
\rho=-i l \rho_{0} \frac{N^{2}}{g \tau}\left(\frac{C_{1}}{k_{2}} \tau^{k_{1}}+\frac{C_{2}}{k_{1}} \tau^{k_{2}}\right) .
$$

Thus, the perturbation of fluid density grows slowly in time as $\tau^{\kappa}$ (see also Ref. [17]), where

$$
\kappa=-\frac{1}{2}+\left[\frac{1}{4}+\left(\frac{\gamma}{v_{0}^{\prime}(\eta)}\right)^{2}\right]^{1 / 2} .
$$

Such a dramatic difference between the time dependencies of the stream function and the density perturbations in a linear system is strictly a nonmodal effect arising from the velocity shear.

It is interesting to note that in convective coordinates (5), spatial derivatives in $\eta$ are absent in the equation for $\zeta_{0}$ and the spatial variable $\eta$ only enters into Eq. (16) and into its solutions (17) and (19) as a parameter. The specific spatial $\eta$ dependence of the solutions (17) and (19) is determined entirely by the $\eta$ dependence of the flow velocity $v_{0}(\eta)$ and by the initial conditions through which the functions $C_{1,2}(l, \eta)$ are determined. It is important to note that the condition $\left|v_{0}^{\prime}(\eta)\right| T>\max \left[(l h)^{-1}\right]$ in Eq. (11) violates near the boundary, where the spatial scale $h$ of the perturbations along the flow shear tends to zero. Therefore, Eqs. (17) and (19) are actually asymptotic solutions, which are valid for times $\tau$ $\geqslant\left[v_{0}^{\prime}(\eta) T\right]^{-1}$ and for locations far from the boundaries, where the conditions given by Eq. (11) or Eq. (12) are valid. For the solution of specific initial and boundary problems, the obtained solutions here would have to be matched with the solutions for $\psi(\tau, l, \eta)$, obtained for the times $\tau$ $\ll\left[v_{0}^{\prime}(\eta) T\right]^{-1}$ and near the boundaries. However, the solution of the complete problem, which includes the initial and boundary problem, is beyond the scope of this work. Here, only the effect of the inhomogeneously sheared flow on the stability of the stratified fluid, i.e., the unbounded case, is considered.

Turning to the next term in the series according to Eq. (15), the equation for $\zeta_{1}(\tau, l, \eta)$ is given by

$$
\begin{aligned}
& \frac{\partial^{2} \zeta_{1}(\tau, l, \eta)}{\partial \tau^{2}}+J(\eta) \frac{1}{\tau^{2}} \zeta_{1}(\tau, l, \eta) \\
& =3 i \frac{v_{0}^{\prime \prime}}{l v_{0}^{\prime}} \frac{1}{\tau} \frac{\partial^{2} \zeta_{0}}{\partial \tau^{2}}-7 i \frac{v_{0}^{\prime \prime}}{l v_{0}^{\prime}} \frac{1}{\tau^{2}} \frac{\partial \zeta_{0}}{\partial \tau}+8 i \frac{v_{0}^{\prime \prime}}{l v_{0}^{\prime}} \frac{1}{\tau^{3}} \zeta_{0}(\tau, l, \eta) \\
& -\frac{2 i}{l \tau} \frac{\partial^{3} \zeta_{0}}{\partial \tau^{2} \partial \eta}+\frac{4 i}{l \tau^{2}} \frac{\partial^{2} \zeta_{0}}{\partial \tau \partial \eta}-\frac{4 i}{l \tau^{3}} \frac{\partial \zeta_{0}}{\partial \eta} .
\end{aligned}
$$

In that equation, the derivatives of $\zeta_{1}$ over $\eta$ are also absent and Eq. (21) is also the ordinary differential equation. The solution to Eq. (21) is readily obtained and is equal to

$$
\begin{aligned}
\zeta_{1}(\tau, l, \eta)= & C_{1}(l, \eta) \frac{\tau^{k_{1}-1}}{l\left(k_{1}-k_{2}\right)}\left[-\frac{i v_{0}^{\prime \prime}(\eta)}{\left[v_{0}^{\prime}(\eta)\right]^{2}}\left(3 k_{1}^{2}-10 k_{1}+8\right)+\frac{2 i}{v_{0}^{\prime}(\eta)} \frac{\partial k_{1}}{\partial \eta}\left[2 k_{1}-3+\left(k_{1}^{2}-3 k_{1}+2\right)(\ln \tau+1)\right]\right. \\
& \left.+\frac{i}{v_{0}^{\prime}(\eta)} \frac{\partial k_{1}}{\partial \eta} \frac{\left(k_{1}^{2}-3 k_{1}+2\right)}{k_{2}}\left(\ln \tau+\frac{1}{2 k_{2}}\right)+\frac{1}{2 k_{2}}\left(\frac{i v_{0}^{\prime \prime}(\eta)}{\left[v_{0}^{\prime}(\eta)\right]^{2}}\left(3 k_{1}^{2}-10 k_{1}+8\right)-\frac{2 i}{v_{0}^{\prime}(\eta)} \frac{\partial k_{1}}{\partial \eta}\left(2 k_{1}-3\right)\right)\right] \\
& -\frac{i\left(k_{1}^{2}-3 k_{1}+2\right)}{v_{0}^{\prime}(\eta) l k_{2}} \frac{\partial C_{1}}{\partial \eta} \tau^{k_{1}-1}+(1 \leftrightarrow 2)
\end{aligned}
$$

for $J(\eta) \neq 1 / 4$ and

$$
\zeta_{1}(\tau, l, \eta)=\frac{1}{\tau^{1 / 2}} \frac{i l v_{0}^{\prime \prime}(\eta)}{\left[v_{0}^{\prime}(\eta)\right]^{2}} \frac{15}{4}\left[C_{1}(\eta)+C_{2}(\eta)\left(\frac{2}{15}+\ln \tau\right)\right]+\frac{1}{\tau^{1 / 2}} \frac{i l}{v_{0}^{\prime}} \frac{5}{2}\left[\frac{\partial C_{1}}{\partial \eta}+\frac{\partial C_{2}}{\partial \eta}\left(\frac{18}{5}+\ln \tau\right)\right]
$$

for $J(\eta)=1 / 4$. In Eq. (22), the notation $(1 \leftrightarrow 2)$ indicates the additional terms similar to those presented explicitly, but with relabeled suffixes in $C_{i}^{(j)}$ and $k_{i}$ according to $1 \rightarrow 2$ and $2 \rightarrow 1$. Equations (17), (18), (22), and (23) demonstrate that solution $\zeta_{1}(\tau)=O\left[\tau^{-1} \zeta_{0}(\tau) \ln \tau\right]$, i.e., solution $\zeta_{1}(\tau)$ algebraically decreases with time more rapidly under the condition given by Eq. (20) than the solution $\zeta_{0}(\tau)$. Therefore, the solution for the stream function $\psi=\left[v_{0}^{\prime}(\eta) T \tau\right]^{-2}\left[\zeta_{0}(\tau)+\zeta_{1}(\tau)\right]$ is stable in the case of strong monotonic, inhomogeneous, flow shear for which Eq. (12) is satisfied. Perhaps most important is that the stabilization of the Rayleigh-Taylor instability by sheared flow persists even for inhomogeneous flow shear. The complete solution has the same structure of the stream function as obtained (in laboratory coordinates) in Eq. (1.6) of Ref. [11]. However, our solution, as given by Eqs. (22) and (23), provides all the coefficients explicitly and the iterative procedure may be easily continued to the desired order of the parameter $\left[v_{0}^{\prime}(\eta) T\right]^{-1}$. 


\section{WEAK NONLINEAR EVOLUTION OF PERTURBATIONS OF THE FLOW WITH INHOMOGENEOUS SHEAR}

Nonlinear studies of the Rayleigh-Taylor instability have been carried out in recent years by Hassam [16], Mikhailenko et al. [17], and others. Hassam performed a one-dimensional calculation and showed that the RayleighTaylor instability in a magnetized plasma may nonlinearly saturate because of flow shear. The nonmodal approach, applied to the study of the weak nonlinear evolution of the two-dimensional Rayleigh-Taylor instability in plasmas with homogeneous flow shear by Mikhailenko et al. [17], demonstrated that homogeneous flow shear stabilizes not only lin- early unstable two-dimensional perturbations of electrostatic potential, but also the nonlinearly unstable perturbations. Now we consider the effect of weak nonlinear nonmodality on the evolution of perturbation for inhomogeneous, monotonic, shear flow. Here we derive a solution to Eq. (9) in the form of the power series in the nonlinearity parameter $\varepsilon T$,

$$
\begin{aligned}
\zeta(\tau, l, \eta)= & \zeta_{0}(\tau, l, \eta)+(\varepsilon T) \zeta_{(1)}(\tau, l, \eta)+(\varepsilon T)^{2} \zeta_{(2)}(\tau, l, \eta) \\
& +\cdots .
\end{aligned}
$$

In Eq. (24), the solution $\zeta_{0}(\tau, l, \eta)$ is determined by Eq. (17) for $J(\eta) \neq 1 / 4$ and by Eq. (19) for $J(\eta)=1 / 4$. The equation for $\zeta_{(1)}(\tau, l, \eta)$, in which terms of zero order in the parameter $\left(v_{0}^{\prime} T\right)^{-1}$ are included, is

$$
\begin{aligned}
\frac{\partial^{2} \zeta_{(1)}(\tau, l, \eta)}{\partial \tau^{2}}+J(\eta) \frac{1}{\tau^{2}} \zeta_{(1)}(\tau, l, \eta)= & \int d l_{1} \int d l_{2} \delta\left(l-l_{1}-l_{2}\right) 2 \frac{i l_{2}^{2}}{l^{2}} \\
& \times\left\{\frac{\partial}{\partial \tau}\left[-\frac{\partial \psi_{0}\left(\tau, l_{1}, \eta\right)}{\partial \eta} l_{2}\left[v_{0}^{\prime}(\eta) \tau\right]^{2} \psi_{0}\left(\tau, l_{2}, \eta\right)+l_{1} \psi_{0}\left(\tau, l_{1}, \eta\right) \frac{\partial}{\partial \eta}\left\{\left[v_{0}^{\prime}(\eta) \tau\right]^{2} \psi_{0}\left(\tau, l_{2}, \eta\right)\right\}\right]\right\},
\end{aligned}
$$

where $C_{i}^{(j)}=C_{i}\left(l_{j}, \eta\right)(i, j=1,2)$. The solution to Eq. (25) is given by

$$
\begin{aligned}
\zeta_{(1)}(\tau, l, \eta)= & \frac{1}{\left(k_{1}-k_{2}\right)\left[v_{0}^{\prime}(\eta)\right]^{2}} \int d l_{1} \int d l_{2} \delta\left(l-l_{1}-l_{2}\right) \frac{l_{2}^{2}}{l^{2}} \int\left[-\frac{8 i l_{2} v_{0}^{\prime \prime}(\eta)}{v_{0}^{\prime}(\eta)}+2 i \frac{\left(l_{2}-l_{1}\right)}{k_{2}} \frac{\partial k_{1}}{\partial \eta}-4 i\left(l_{2}-l_{1}\right) \frac{\partial k_{1}}{\partial \eta}\left(\ln \tau+\frac{1}{k_{2}}\right)\right] \\
& \times C_{1}^{(1)} C_{1}^{(2)} \tau^{k_{1}-k_{2}}+\left[\frac{4 i l_{2}}{k_{1}} \frac{v_{0}^{\prime \prime}(\eta)}{v_{0}^{\prime}(\eta)}\left(C_{1}^{(1)} C_{2}^{(2)}+C_{2}^{(1)} C_{1}^{(2)}\right)-\frac{2 i l_{2}}{k_{1}}\left(C_{1}^{(2)} C_{2}^{(1)} \frac{\partial k_{2}}{\partial \eta}+C_{1}^{(1)} C_{2}^{(2)} \frac{\partial k_{1}}{\partial \eta}\right)\left(\ln \tau+\frac{k_{2}}{k_{1}}\right)\right. \\
& \left.+\frac{2 i l_{1}}{k_{1}}\left(C_{1}^{(1)} C_{2}^{(2)} \frac{\partial k_{2}}{\partial \eta}+C_{1}^{(2)} C_{2}^{(1)} \frac{\partial k_{1}}{\partial \eta}\right)\left(\ln \tau+\frac{k_{2}}{k_{1}}\right)\right]+\left[\frac{8 i k_{1} l_{2}}{\left(2 k_{1}-k_{2}\right)} \frac{v_{0}^{\prime \prime}(\eta)}{v_{0}^{\prime}(\eta)}+\frac{2 i\left(l_{1}-l_{2}\right)}{\left(3 k_{2}-2\right)} \frac{\partial k_{2}}{\partial \eta}-\frac{\left(4 i l_{2} k_{2}-4 i l_{2}+4 i l_{1} k_{1}\right)}{\left(3 k_{2}-2\right)}\right. \\
& \left.\left.\times\left(\ln \tau-\frac{1}{3 k_{2}-2}\right)\right] C_{2}^{(1)} C_{2}^{(2)} \tau^{k_{2}-k_{1}}\right\}-\frac{2 i}{\left(k_{1}-k_{2}\right)\left[v_{0}^{\prime}(\eta)\right]^{2}} \int d l_{1} \int d l_{2} \delta\left(l-l_{1}-l_{2}\right) \frac{l_{2}^{3}}{l^{2}}\left[2 C_{1}^{(2)} \frac{\partial C_{1}^{(1)}}{\partial \eta} \tau^{k_{1}-k_{2}}\right. \\
& \left.+\left(C_{2}^{(2)} \frac{\partial C_{1}^{(1)}}{\partial \eta}+C_{1}^{(2)} \frac{\partial C_{2}^{(1)}}{\partial \eta}\right) \frac{1}{k_{1}}-2 C_{2}^{(2)} \frac{\partial C_{2}^{(1)}}{\partial \eta} \frac{1}{3 k_{2}-2} \tau^{k_{2}-k_{1}}\right]+\frac{2 i}{\left(k_{1}-k_{2}\right)\left(v_{0}^{\prime}(\eta)\right)^{2}} \int d l_{1} \int d l_{2} \delta\left(l-l_{1}-l_{2}\right) \frac{l_{1} l_{2}^{2}}{l^{2}} \\
& \times\left[2 C_{1}^{(1)} \frac{\partial C_{1}^{(2)}}{\partial \eta} \tau^{k_{1}-k_{2}}+\left(C_{1}^{(1)} \frac{\partial C_{2}^{(1)}}{\partial \eta}+C_{2}^{(1)} \frac{\partial C_{1}^{(2)}}{\partial \eta}\right) \frac{1}{k_{1}}-2 C_{2}^{(1)} \frac{\partial C_{2}^{(2)}}{\partial \eta} \frac{1}{\left(3 k_{2}-2\right)} \tau^{k_{2}-k_{1}}\right]+(1 \leftrightarrow 2)
\end{aligned}
$$

for $J(\eta) \neq 1 / 4$. In Eq. (26), the notation $(1 \leftrightarrow 2)$ indicates additional terms similar to the terms presented explicitly, but with relabeled suffixes, $i=1,2$ in $C_{i}^{(j)}$ and $k_{i}$ according to $i=1$ is changed to $i=2$, and $i=2$ is changed to $i=1$. For the values of $\eta$ for which $J(\eta)=1 / 4, \zeta_{(1)}(\tau, l, \eta)$ is given by

$$
\begin{aligned}
\zeta_{(1)}(\tau, l, \eta)= & \frac{4 i}{l^{2}\left[v_{0}^{\prime}(\eta)\right]^{2}} \int d l_{1} \int d l_{2} \delta\left(l-l_{1}-l_{2}\right) l_{2}^{2}\left\{l _ { 2 } \left[\left(3 \frac{\partial C_{2}^{(1)}}{\partial \eta} C_{1}^{(2)}+16 \frac{\partial C_{2}^{(1)}}{\partial \eta} C_{2}^{(2)}+\frac{\partial C_{1}^{(1)}}{\partial \eta}\left(C_{1}^{(2)}+3 C_{2}^{(2)}\right)\right)\right.\right. \\
& \left.+\ln \tau\left(\frac{\partial C_{2}^{(1)}}{\partial \eta} C_{1}^{(2)}+\frac{\partial C_{1}^{(1)}}{\partial \eta} C_{2}^{(2)}+6 \frac{\partial C_{2}^{(1)}}{\partial \eta} C_{2}^{(2)}\right)+\ln ^{2} \tau \frac{\partial C_{2}^{(1)}}{\partial \eta} C_{2}^{(2)}\right]+l_{1}\left[\left(3 \frac{\partial C_{2}^{(2)}}{\partial \eta} C_{1}^{(1)}+16 \frac{\partial C_{2}^{(2)}}{\partial \eta} C_{2}^{(1)}\right.\right. \\
& \left.\left.+\frac{\partial C_{1}^{(2)}}{\partial \eta}\left(C_{1}^{(1)}+3 C_{2}^{(1)}\right)\right)+\ln \tau\left(\frac{\partial C_{2}^{(2)}}{\partial \eta} C_{1}^{(1)}+\frac{\partial C_{1}^{(2)}}{\partial \eta} C_{2}^{(1)}+6 \frac{\partial C_{2}^{(2)}}{\partial \eta} C_{2}^{(1)}\right)+\ln { }^{2} \tau \frac{\partial C_{2}^{(2)}}{\partial \eta} C_{2}^{(1)}\right] \\
& \left.-\frac{v_{0}^{\prime \prime}(\eta)}{v_{0}^{\prime}(\eta)} l_{2}\left\{\left[3 C_{2}^{(1)} C_{1}^{(2)}+16 C_{2}^{(1)} C_{2}^{(2)}+C_{1}^{(1)}\left(C_{1}^{(2)}+3 C_{2}^{(2)}\right)\right]+\ln \tau\left(C_{2}^{(1)} C_{1}^{(2)}+C_{1}^{(1)} C_{2}^{(2)}+6 C_{2}^{(1)} C_{2}^{(2)}\right)+\ln ^{2} \tau C_{2}^{(1)} C_{2}^{(2)}\right\}\right\} .
\end{aligned}
$$


Both expressions for $\zeta_{1}(\tau)$ confirm that weak, nonmodal nonlinearity does not eliminate the stabilization of the Rayleigh-Taylor instability by inhomogeneous, monotonic shear flow that satisfies the constraint described by Eq. (12). The solutions for the nonlinear nonmodal perturbation of the stream function $\psi_{(1)}(\tau, l, \eta)=\left[v_{0}^{\prime}(\eta) T \tau\right]^{-2} \zeta_{(1)}(\tau)$ with either $J(\eta) \neq 1 / 4$ or $J(\eta)=1 / 4$ will decrease with time provided that

$$
\left|v_{0}^{\prime}(\eta)\right| \geqslant \frac{2}{\sqrt{3}} \gamma
$$

Otherwise the perturbations will grow algebraically. The constraint described by Eq. (28) is more restrictive than the constraint given by Eq. (20) for the linear stabilization of the stream function because in the range $(1 / \sqrt{2}) \gamma<\left|v_{0}^{\prime}(\eta)\right|$ $<(2 / \sqrt{3}) \gamma$, nonlinearly excited perturbations of the stream function $\psi_{1}$ will grow algebraically.

It is interesting to note that the condition (28) was obtained earlier in Ref. [17] for the case of homogeneous flow shear, even though the nonlinear (right side) of the equation for $\zeta_{(1)}$ was different in the cases of homogeneous and inhomogeneous flow shear. The only differences in time dependencies in the case of inhomogeneous shear are the appearance of $\ln \tau$ and $(\ln \tau)^{2}$ multipliers in Eqs. (26) and (27), because of the $\eta$ dependencies of $k_{1,2}$. However, these multiplicative factors do not affect the decaying nature of the solutions for $\psi_{(1)}=\left[v_{0}^{\prime}(\eta) T \tau\right]^{-2} \zeta_{(1)}(\tau)$ under the constraint of Eq. (28).

\section{CONCLUSIONS}

In this work, we have developed an analytical framework that is a natural extension of the nonmodal approach for homogeneous sheared flows to the case of inhomogeneous shear. The linear and weak nonlinear evolution of perturbations in systems with monotonic, inhomogeneous shear was determined and it was demonstrated that stabilization of the Rayleigh-Taylor instability by shear flow can persist in the case of inhomogeneously sheared flow. The temporal evolution of the initial perturbations depends on the relative mag- nitudes of the shear parameter, $v_{0}^{\prime}(\eta) T$, and the nonlinearity parameter, $\varepsilon T$. In the specific case of monotonic shear, sheared flow disturbances that satisfy Eq. (12) for all values of $\eta$ are stable to the development of Rayleigh-Taylor instability. The stabilization arises from nonmodal effects due to the flow shear.

Stabilization of the Rayleigh-Taylor instability in plasmas and in ordinary, homogeneously sheared, flows was reexamined in a recent paper by Benilov et al. [19]. They concluded that stabilization of the Rayleigh-Taylor instability by homogeneous shear flow is a "quirk" of the model chosen and any deviation from a linear velocity profile triggers instability, i.e., inhomogeneous shear is not stabilizing. Their conclusion was based on a study of the stability of small wave number, $l$, perturbations in systems with monotonic shear flow and a study of the stability of jet flow in the vicinity of a tip where $v_{0}^{\prime}\left(\eta_{0}\right)=0$, i.e., the cases were considered in which shear flow effects are too small (as in the case of small $l$ ) or even absent (as at the tip of the jet flow). However, it is important to note that the stabilization of the Rayleigh-Taylor instability even by homogeneous shear flow is not a universal effect for all values of the flow shear $v_{0}^{\prime}$ and wave number $l$. Stabilization by homogeneous flow shear requires fulfillment of the condition $\left|v_{0}^{\prime}(z)\right| \geqslant 2^{-1 / 2} \gamma$ for linearly unstable perturbations and fulfillment of slightly more stringent conditions for nonlinearly unstable perturbations [17]. In fact, homogeneous shear flow provides no stabilizing effect for perturbations with $l$ $=0$ [17]. The stabilization condition found in this work, Eq. (12), is a similar constraint for inhomogeneously sheared flow. Our analysis is consistent with the numerical results described in Ref. [19] in that for cases where the effect of flow shear, as expressed through the nonmodal terms in Eq. (6) or Eq. (9), is minimal or absent (as it is in the two cases examined in Ref. [19]), the Rayleigh-Taylor instability grows. However, if the perturbation characteristics and flow shear satisfy Eq. (12), all perturbations of the stream function are stable.

\section{ACKNOWLEDGEMENTS}

V.S.M. wishes to thank Professor A.B. Hassam for drawing attention to the paper by Benilov et al.
[1] S. Chandresekhar, Hydrodynamic and Hydromagnetic Stability, Int. Ser. Monographs on Physics (Clarendon Press, Oxford, 1961).

[2] R. D. Hazeltine and J. D. Meiss, Plasma Confinement, Ser. Monographs Frontiers in Physics (Addison-Wesley/Publ. Company, Reading, MA, 1992).

[3] S. L. Ossakow, J. Atmos. Terr. Phys. 43, 437 (1981).

[4] B. G. Fejer and M. C. Kelley, Rev. Geophys. Space Phys. 18, 401 (1980)

[5] J. W. Miles, J. Fluid Mech. 10, 496 (1961).

[6] H. L. Kuo, Phys. Fluids 6, 195 (1963).

[7] P. N. Guzdar, P. Satyanarayana, J. D. Hyba, and S. L. Ossa- kow, Geophys. Res. Lett. 9, 547 (1982).

[8] K. M. Case, Phys. Fluids 3, 144 (1960).

[9] R. E. Kelly and S. A. Maslowe, Stud. Appl. Math. 49, 301 (1970).

[10] J. R. Booker and F. P. Bretherton, J. Fluid Mech. 27, 513 (1967).

[11] S. N. Brown and K. Stewartson, J. Fluid Mech. 100, 811 (1980).

[12] L. N. Trefethen, A. E. Trefethen, S. C. Reddy, and T. A. Driscoll, Science 261, 578 (1993).

[13] R. J. Hartman, J. Fluid Mech. 71, 89 (1975).

[14] Lord Kelvin, Philos. Mag. 24, 188 (1887). 
[15] O. M. Phillips, The Dynamics of the Upper Ocean (Cambridge University Press, Cambridge, 1966).

[16] A. B. Hassam, Phys. Fluids B 4, 485 (1992).

[17] V. S. Mikhailenko, V. V. Mikhailenko, and J. Weiland, Phys. Plasmas 9, 2891 (2002).
[18] V. S. Mikhailenko, V. V. Mikhailenko, M. F. Heyn, and S. M. Mahajan, Phys. Rev. E 66, 066409 (2002).

[19] E. S. Benilov, V. Naulin, and J. Juul Rasmussen, Phys. Fluids 14, 1674 (2002). 